\title{
A Semantic-based Approach to Improve Knowledge Intensive Service Processes
}

\author{
Bernd Stieger \\ ABB Power Products \\ Service
}

\author{
Markus Aleksy \\ $A B B$ Corporate Research \\ Industrial Software and Application
}

\begin{abstract}
The industrial service business requires much information about the application area, involved products, their configurations and dependencies as well as many other types of information, thus is considered being very knowledge intensive. A lot of the required information is already available in various enterprise information systems that are managed within the organization. However, they consist of a mixture of structured and unstructured information contained in various data sources. Thus, it is challenging to extract and combine the right pieces of information in order to provide valuable knowledge to involved participants in service processes. This paper presents how semantic web technologies can be utilized in order to support industrial service use cases. After presenting the prerequisite tasks that include activities such as identifying processes, describing roles, and collecting requirements, a technical framework is presented and discussed. Finally, the evaluation of the developed knowledge portal prototype is discussed.
\end{abstract}

\section{Introduction}

The importance of the service sector in already industrialized countries is higher than ever before. In Germany and the United States the services amount regarding the GDP in the year 2010 counts for $71.3 \%$ and $76.7 \%$, respectively, whereas the contribution of the industrial and agricultural sectors to the GDP is less than 30 percent [17]. Lovelock and Gummesson (2004) characterize services according to the IHIP principle that means that services are Intangible, Heterogeneous, Inseparable and Perishable [11]. Intangible refers to the fact that services are not physical; they cannot be touched or felt.

They can be described as any act of performance offered by one party to another [10]. They are heterogeneous since they are usually highly customized. The inseparability refers to the coproduction of services where both producer and consumer are involved. Perishable means that it is not possible to store services. However, it is possible to store information about services. The IHIP properties can be easily applied to an industrial service context where performing service tasks will be more complex since the products are becoming technically more and more advanced, e.g., because of the miniaturization or the increasing amount of embedded software. Thus, in order to address customers' demands more knowledge is necessary within service delivery processes. On the other hand, there is already plenty of raw data and information available in today's enterprises systems which need to manage terabytes of data which are stored in heterogeneous information systems.

In order to find the right information at the right time, a search over several data sources has to be performed. Very often relevant service data sources are not integrated and they need to be searched consecutively when looking for a piece of information. This is in general a very time-intensive task since one has to know which are the most likely data sources to find the required information and how to perform searches in every system. Furthermore, the costs of using and federating data from traditional integrated data sources are becoming higher with the increasing number of data sources. [15] shows the increasing cost of using data sources in relation with a higher number of these sources, whereas when taking concepts from the semantic web, e.g., ontologies, into consideration these costs could be lowered.

The research project Aletheia is investigating how semantic web technologies and concepts ([4],[16],[6]) can be utilized to support knowledge intensive service processes [3]. The discussed approach focuses on gaining knowledge from different sources, structured and unstructured ones, the derivation of implicit knowledge and a uniform representation of the knowledge. Section 2 describes basic scenarios and service process actors as well as most important requirements. In section 3 there is a discussion of the technological realization.

Evaluation and test results are presented in section 4. Section 5 covers relevant related work and section 6 offers some concluding remarks.

\section{Service Scenario and Requirements}

In a first step we evaluated the existing industrial service processes, identified the potential roles (user profiles) and suitable application scenarios that may benefit from semantic integration of heterogeneous information sources and knowledge support. Here, 
the following user profiles could be identified: Service technicians, experienced service planners, service experts, inexperienced service engineers, call center agents, product managers, service partners, plant managers, and technical consultants.

The identified application scenarios that provide a major opportunity for improvement are related to contact center support, support for remote service tasks, and field service support. In the following section, we will discuss the corresponding use cases.

\subsection{Exemplary Industrial Service Process}

In order to derive an appropriate knowledge management strategy for the service business unit and the processes, we followed the knowledge strategy process approach suggested by van der Spek and Spijkervet [18]. At first, business unit goals and core processes in order to reach these goals need to be identified. Additionally, it will be highlighted which process steps are the most knowledgeintensive ones and an overview about knowledge areas will be given.

The following goals were stated as the most important by the service business unit:

(i) Increase of customer satisfaction

(ii)Efficient and effective service delivery processes

(iii) Service unit growth in terms of number of people and revenues

In order to draw near the goal of increasing the customer satisfaction, the most important core process is the 'Field Service Job' since the activities performed by the engineers at the customer sites are crucial for the contentment of the customer. An efficient and effective service delivery process can be reached with paying attention to the sub-processes 'Dispatching of Personnel and Material' as well as again 'Field Service Job'. For the last goal 'service unit growth' it is vital to perform more service jobs with the current service engineers and to increase the number of service jobs even more with hiring new service engineers. As a result, the core process 'Field

Service Job' gains even more importance, since current engineers must be well prepared to execute their knowledge-intensive jobs faster and new employees need to be equipped with service job related knowledge.

In general, a business process analysis can be used in order identify knowledge-intensive process parts.

The business processes analysis was conducted together with service operations staff. Every process part has been modeled with using process diagrams which were easily understandable by the interview participants during the verification of the recorded processes. One of the most important service process actors in the service delivery process is the service engineer who has most of the direct contact with the customers. Usually, it is the responsibility of the engineers to prepare themselves prior to the actual work at an object location. The required information consists, for example, of details about the customer's problem and the installed base history. Figure 1 presents a standard service process which consists of six steps, namely: handling customer's request, processing service notification, dispatching of service engineers and required spare parts, executing the field service job, recording of the activity performed by the service engineer and, finally, invoicing the customer.

\subsection{Use Case Overview}

The ultimate goal of utilizing semantic web technologies is the support of service process actors in performing their knowledge intensive tasks. Figure 2 depicts the most important actors in service processes and service data sources which provide information necessary for certain process steps. There are contact center agents who have the first

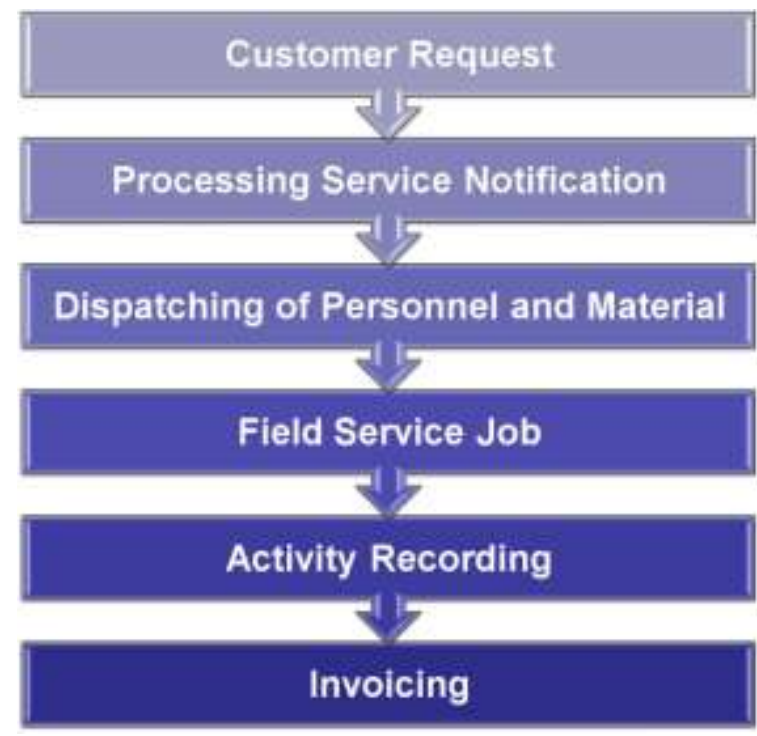

Figure. 1. Typical Service Process

contact to customers who usually report a problem or request certain service activities, e.g., provision of product manuals, maintenance instructions or ordering a service engineer for an on-site visit.

The challenge for these agents is an instant reaction with the right replies to the customers' requests which are often interlinked with performing a search over data sources which contain product documentation, service people, and customer information. The relevant data sources for this process step are file servers, the Intranet, or local 


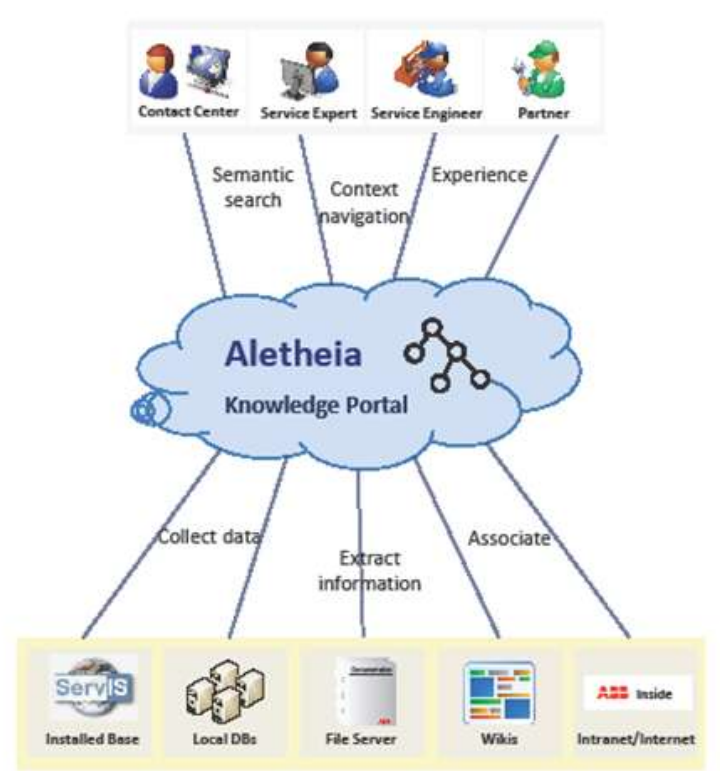

Figure 2. Service actors and relevant data sources

databases. Secondly, there are remote service experts who are responsible for solving an actual customer problem over the phone with using their expert knowledge. However, in order to understand the problem context in detail they also need to find information about the customers' product history, installations, configurations, specific customizations and so on and so forth. They perform searches in installed base systems, which contain installed products and systems at customer sites, and also local databases and Wikis where standard configurations and best practices are being stored.

Service engineers are responsible for performing service jobs at customer sites. They depend on information from all of the in Figure 2 presented data sources in order to prepare their job. Furthermore, third party service partners are being utilized for performing service jobs for locations that lack of local service centers or these centers are too far away from the concerning customer sites. They require an even better preparation and there is a need to provide them standardized service procedure guidelines as well as more detailed product and system manuals. Every mentioned actor depends on information from different data sources which need to be investigated during each task, and the underlying searches consume a huge amount of time. In order to optimize these search and information provision processes, requirements regarding a desired knowledge portal where specified. These will be presented in the following subsection.

\subsection{Requirements}

Based on the general scenario descriptions about 60 requirements could be derived and identified during workshop discussions with potential endusers whereas the most important ones are presented in this paper. For structuring purposes the following classes were chosen:

- Input Capabilities

Entering a problem or specifying a query should be easy for the users. A simple input form should be provided to enter search queries. Furthermore, an important user requirement was the provision of automatic word extensions in order to lower the effort for typing long and more difficult to spell product names or error codes. Both possibilities, to enter keywords or describing the problem as a story should be supported by the knowledge portal.

- Search and Output Capabilities

In the area of search requirements which are closely linked to the previously discussed input capabilities, the main focus was on providing intelligent search mechanisms. Standard queries should be considered in terms of trying to interpret certain keywords and their relation among each other. For example, if entering a query of the type $\langle$ ProductType $\rangle\langle$ Role $\rangle$ which could look like '800xA Expert' the user expects to receive a list of experts for the product type ' $800 \mathrm{xA}$ ' and does not want to have search results which match only the strings ' $800 \times \mathrm{xA}^{\prime}$ and 'Expert'. Further standard queries such as <ProductType> <Application> $<$ ServiceReport $>$ should also be understood by the system in a way, that the user is interested in service reports which belong to installations of a certain product type and additionally, these installations belong to an application and are linked to a service report. Graphical result presentations should be realized to enable users to explore and navigate through the knowledge space defined by semantic relations between entities and concepts. Especially in the area of unstructured information the results should be generated based on extracted information out of relevant documents in contrast to displaying a plain list of document links. There were also more elementary requirements such as including search constraints on data sources to include, ranking and sorting of search results as well as topic classification of search results.

- Knowledge Contribution Facility

For users it must be possible at any time to add new knowledge assets to the knowledge base, e.g., best practices or lessons learned from certain customer problem reports and their solution. For this functionality dynamic templates should be provided in order to capture vital parts of the knowledge contribution. In addition, when adding documents via the knowledge portal, it must be possible to add specific tags in terms of 
meta-data which will support the later retrieval. Possibility to tag search results should be also provided by the portal and additionally, these tags should be considered again for later search processes.

- User Context-related Requirements

The main user interface requirement is the provision of a web front-end which can be accessed by common browsers and no installation routine is necessary. Another requirement concerns more the device, since service engineers would prefer mobile smart phones to work with and thus, the knowledge portal should be accessible with regard to adapting the application to make it fit to smaller screens and less processing power. In general, the portal should be configurable according to users' needs. As already mentioned there are requirements regarding data input forms and visualization in the categories input and output capabilities. The users' context needs to be considered in terms of service unit where engineers belong to or grade of experience whereupon this context information should be considered when generating search results.

- Data Source Integration

An integration of existing data sources is one of the most central requirements since these sources are filled by usage of service applications and systems and their contained information is vital for re-usage. It can be distinguished between structured and unstructured information. Structured information can be found in databases such as in an installed base system or other local databases based on, e.g., Oracle or MS-SQL. Unstructured information plays an important role which can be found on file servers, Wikis or Intranet/Internet. Examples are MS Office documents, PDFs, HTML-websites or plain text documents which have either no structure at all or only a weak structure incorporated.

\section{Technical Framework and Prototype}

In this section, there are initial technical discussions regarding the architecture and implemented components as well as some search examples are used to illustrate how the presented requirements were realized in the knowledge portal prototype.

\subsection{Architecture}

For accessing knowledge which is stored in heterogeneous back-end systems, it is necessary to provide an appropriate access for the users to the knowledge portal where they perform their search, e.g., a web frontend. The proposed architecture of the system consists of 3-tiers ([21], [14], [20]). Figure 3 shows the client tier, business logic tier, and the data tier. The client tier comprises a variety of usable devices such as smart phones, laptops or Desktop PCs. The service hub, main part within the business logic tier, is the central component of the whole architecture. It is connected to the systems of the data tier and provides functionalities, e.g., search services, data source connection, and access services. The service hub stores and manages the ontology, and comprises repositories for crawled and indexed documents as well as facts and metadata. There is a

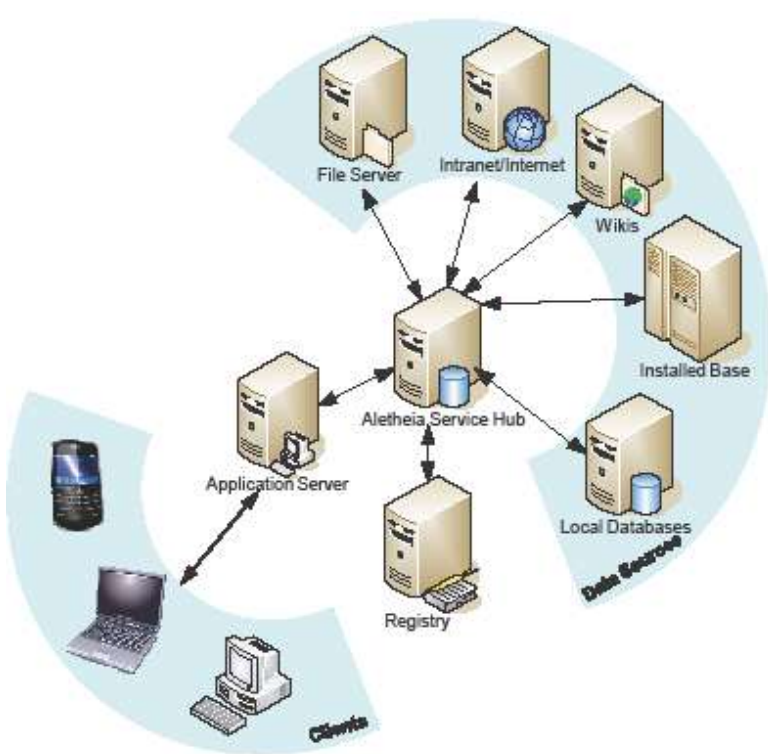

Figure 3. Aletheia knowledge portal architecture (based on [1])

semantic repository with an index built from structured data from databases and an uncertain repository which stores crawled data from unstructured sources and the corresponding index. In addition, it is responsible for inter domain communication with other optional service hubs. The registry, connected to the service hub, facilitates the configuration of external applications, data sources, and service hub components. The application server is a host of web-based applications and is responsible for processing information that is made available by the service hub and sending them to the clients. The data tier comprises various information systems and data sources which were discussed in section 2 . 


\subsection{Semantic Search and Service Ontology}

The actual semantic search is based on an ontology that has been developed for the service area. The ontology contains concepts and relations which describe the application domain, in our case the industrial service domain. A common understanding among different people working in the same domain can be supported with using ontological descriptions. A huge advantage of ontologies is the fact that they are machine readable and -interpretable. The used language is formalized and there are standardized ontology languages available such as OWL or RDFS [19]. In Figure 3 there is a snippet of our service ontology contained which shows different important concepts and their relation to each other. In the example ontology it can be seen that there are installations and their relation to product types of relevance, furthermore, service jobs are related to installations and these are performed on the other hand by service engineers who write service reports. Based on the concepts and their relations among each other, certain facts can be derived, e.g., when service engineers perform service jobs on an installation of a product type, it can be concluded that these engineers are experts for these product types. Additional important concepts are service contracts, customers, configurations as well as error codes that can be used to further describe the service domain. This ontology is stored as structured and machine-readable code in the service hub (cp. Figure 4). For users in a service office, such as contact center agents or remote experts, there is a possibility to use a web frontend since no installation is required and it can be accessed from every desktop computer. When performing a semantic search, the ontology is utilized to support the users, e.g., with using auto-completion based on terms stored in the ontology. Thus, it is possible that the entered keywords (cp. Figure 4) are actually interpreted by the Aletheia system, e.g., 'AC800M' as a control system product, 'Chemical' as a branch and 'error 3' as an error code of a service report. That means, the search result has been concluded in a way that the three terms have been interpreted and assigned to their underlying concept. In addition, valid relations for the three terms have been found, and their relation to each other is shown in the semantic graph in Figure 4. It can be read that there is an existing installation 'AC800M Installation - 3.2004..' that has the product type 'AC800M', this installation is located at a customer's site operating in the branch 'Chemical', and for this installation exist a service job with a service report containing 'error 3'. It must be noted that the search terms do not have to be contained in the search result itself, but the search result relates clearly to the search terms brought into relation to each other. This example shows also the
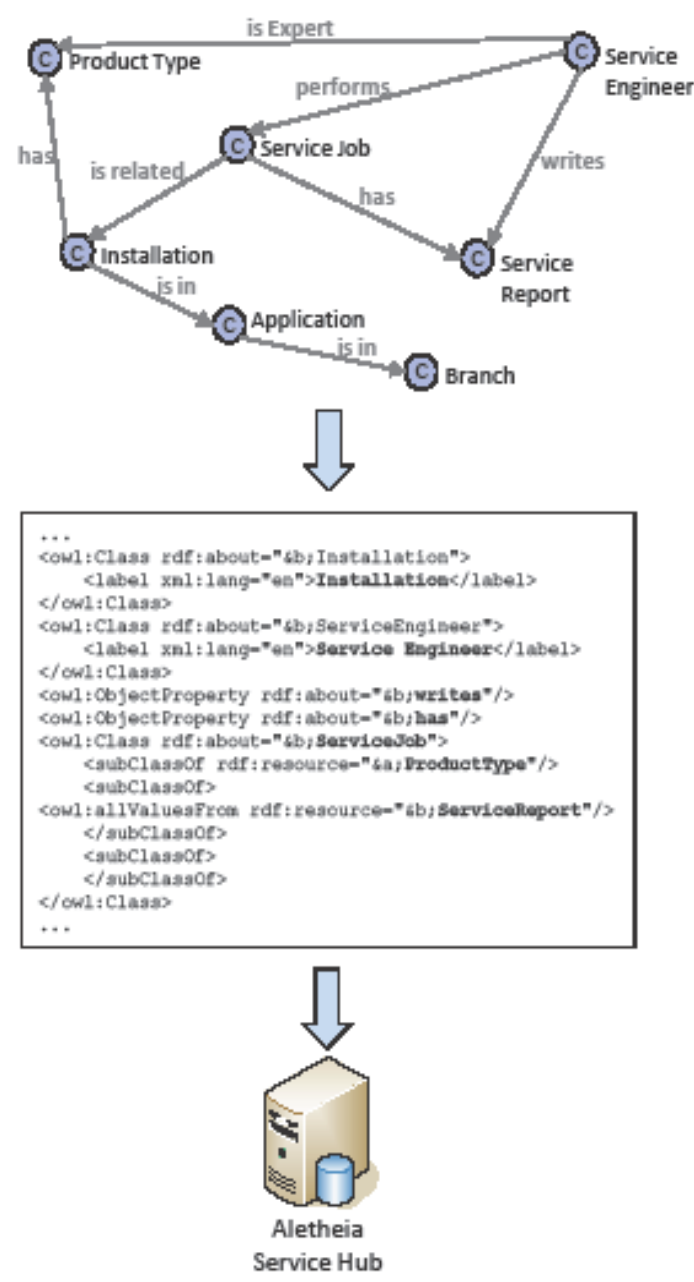

Figure 4. Ontology modeling and machine-
code

two most important search functionalities, first the semantic search which is basically an intelligent keyword search and secondly, an explorative search in terms of navigating in semantic graphs (cf. Figure 5). In order to support service engineers and service partners which are mobile field workers it is necessary to provide them mobile knowledge solutions. Thus, a thin client application for Android OS has been developed that can access all services provided by the Aletheia service hub (cp. section 2.1). The same query as mentioned above, namely 'AC800M Chemical error 3', can be entered on a mobile device, e.g., smart phone. Figure 6 presents the succeeding steps which need to be performed by a user to finally receive a result. The two screen shots show how the semantic auto-completion supports users in choosing the appropriate search term. For instance, the term 'Chemical' can be disambiguated by the user in a way that he can declare what he means, whether he means 'Chemical' as a branch or as an specialized application type. Figure 7 shows the overall results 


\section{Problem:}
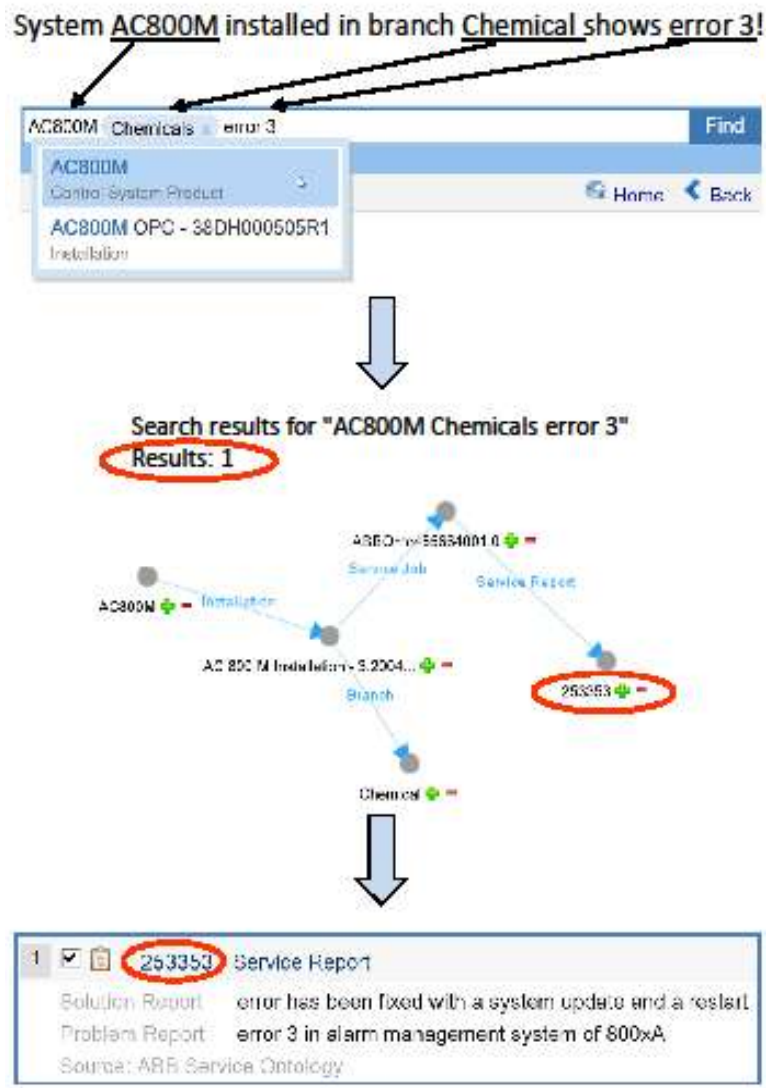

Figure 5. Example of a semantic search with a webbased knowledge portal

for this query and the details of the result service report. The main challenge in the user interface design for this application was dealing with small screens and limited input capabilities of mobile devices without abandoning the required search functionalities [2].

\section{Evaluation and Test Results}

Different aspects of the knowledge portal have been evaluated. Among the five test areas were installation-, user interface-, functionality-, search quality- and performance tests. Since the evaluation regarding search quality and performance was considered as most important and these areas showed the highest improvement potential at the beginning of the project, the focus in this section is on these test results. The results for installation-, user interfaceand functionality tests were quite good and the test users had only minor change and improvement suggestions. In order to measure the search quality degree, it is possible to use 'precision' and 'recall' that are mostly used in the area of information retrieval evaluation ([13], [12]). Both values can be defined with using numbers of retrieved documents and relevant documents. The number of retrieved documents stands for all results that are received for a search query. The relevant documents are a number of documents which are expected or needed in a result list by a user who is searching in a set of documents. To determine which documents are relevant for a certain search query it is necessary to manually decide for each document whether it is relevant or non-relevant. After performing these decisions the received result is called gold standard or ground truth for judging the relevance [13]. For the three data sources 'File Server', 'Wiki' and 'Intranet' such a gold standard has been created for 35 typical search queries. Test samples were extracted from these data sources and the overall document corpus contained about 7000 documents. In general, the 'precision' is defined as the quota of number of documents which are relevant for the search considering all retrieved documents (cp. 1):

$$
\text { precision }=\frac{\mid\{\text { relevant docs }\} \cap\{\text { retrieved docs }\} \mid}{\mid\{\text { retrieved docs }\} \mid}
$$

'Recall' stands for the portion of documents which are relevant to the search query and are listed in the result set (cp. 2):

$$
\text { recall }=\frac{\mid\{\text { relevant docs }\} \cap\{\text { retrieved docs }\} \mid}{\mid\{\text { relevant docs }\} \mid}
$$

Based on the fact that it is quite trivial to receive always a value of $100 \%$ for the 'recall' value, since it is possible to deliver all available documents to every search query, it is necessary to consider the values for 'precision' and 'recall' always together. For this purpose, the so called F1-Score or Fmeasure can be used to express the weighted average of the 'precision' and 'recall' [13]. The formula is defined as (cp. 3):

$$
F=2 * \frac{\text { precision } * \text { recall }}{\text { precision }+ \text { recall }}
$$

If expressed in percentages the worst value for the F-measure is $0 \%$ and the best value possible $100 \%$. Table 1 presents four test queries that were used to calculate number for recall, precision and the Fmeasure. As mentioned beforehand, it is rather trivial to receive a recall of $100 \%$ as it is proven in the table. However, the best insights can be derived from the F-measure which is quite good for the stated queries. Since 35 queries were used to create a gold 
Table 1. Example of Four Queries as Part of the Recall, Precision, and F-Measure

\begin{tabular}{|l|c|c|c|c|}
\hline Query & $\begin{array}{c}\text { IRB6400M98 } \\
\text { manipulator }\end{array}$ & FXE4000 & $\begin{array}{c}\text { FXE4000 } \\
\text { Configuration }\end{array}$ & $\begin{array}{c}\text { FXE4000 } \\
\text { Application }\end{array}$ \\
\hline \# of relevant docs & 11 & 18 & 4 & 4 \\
\hline \# of retrieved docs & 18 & 26 & 4 & 5 \\
\hline \# of relevant docs found & 11 & 18 & 4 & 4 \\
\hline Recall & 1 & 1 & 1 & 1 \\
\hline Precision & 0.611111111 & 0.692307692 & 1 & 0.8 \\
\hline F-measure & 0.75862069 & 0,818181818 & 1 & 0.888888889
\end{tabular}

standard, the above stated three metrics were calculated for all the queries. Considering these 35 queries the weighted average for the recall is $100 \%$, the average precision is $82.68 \%$ and the average value for the F-measure is $88.98 \%$. In order to measure the performance of the knowledge portal prototype 25 typical user queries were used and each execution time has been recorded. A rough target for the answering time was 3000 milliseconds per query. The performance tests have been performed on a server with $2.53 \mathrm{GHz}$ and 4 processors, $10 \mathrm{~GB}$ physical RAM and a 64-bit Windows Server operating system. The prototype used Apache Tomcat as a web server and the underlying Java Virtual Machine that was executed utilized 6 GB of heap memory. Queries with different complexity degrees were chosen to investigate how the system is behaving under various reasoning and answering conditions. Table 2 presents the test queries and their corresponding query type. 'Instance' stands for a term which has been recognized by the autocompletion function and thus, it means this term exists as a value among the datasets. 'Concept' stands for a class contained in the ontology which contains several instances. Typical concepts were discussed in section 2.2, e.g., configuration, product type or service engineers. A term is expressed as 'string' which might be somewhere contained in a dataset but is not recognized as an 'Instance' as part of the auto-completion. Taking into consideration that each query can be combined based on these three types, the complexity might be different and thus, the reasoning process to generate a result might take longer. About 40,000 datasets were used for the performance evaluation. In Figure 7 the evaluation results are displayed and it can be recognized that the answering time for the queries 22-25 is longer since four terms had been used and the processing of the interpretation of their relation to each other as well as the reasoning of valid results is more complex. The reason for a distinguishable longer answering time of the queries 4, 5 and 21 from the answering times of the other queries is the fact that the concepts product type as well as service report contain much more instances than other concepts. Again, in order to process a higher number of instances to answer a query leads to an overall longer execution time. Taking all evaluation results into consideration it can be stated that the users were quite satisfied with the knowledge portal since it showed an overall improvement compared to the systems and applications that are available in today's IT landscape.

\section{Related Work}

Semantic web technologies and concepts are meanwhile well established in the research community and a great deal of publications exists. Thus, only some articles with a closer relationship to this paper will be named as related work since there is a myriad of contributions which are important but rather distantly related. Demartini discusses the applicability of semantic technologies for enterprise search on a very high level and gives recommendation which approaches fit to different enterprise search scenarios [6]. In papers by Delgado et al. and Grudin the focus is on highlighting the challenges in searching enterprise system where mostly structured and unstructured information is being stored [5], [8]. Especially Grudin points out that it is quite difficult to find documents in enterprise systems since often the context of documents and metadata is missing. He tries to emphasize an awareness that a lot administrative effort is required to create metadata or to create and utilize ontologies. Further success stories and challenges regarding enterprise knowledge management systems are presented in [9]. In the contribution by Fagrell et al. a knowledge management architecture is discusses that focuses on mobility aspects of field workers who need to perform searches while they are out of office [7]. 
Table 2. Queries Used for Performance Measurements

\begin{tabular}{|l|l|l|}
\hline No & Query Type & Query \\
\hline 1 & Instance, string & Multi-FID 14 Ausfall \\
\hline 2 & Instance, Concept & Limas 11 Configuration \\
\hline 3 & Instance, Concept & Advance Cemas FTIR Configuration \\
\hline 4 & Instance, Concept & Chemical Producttype \\
\hline 5 & Instance, Concept & Public Supply and Utilities Producttype \\
\hline 6 & Instance, Concept & Advance Cemas FTIR Expert \\
\hline 7 & Instance, Concept & Limas 11 Expert \\
\hline 8 & Instance, Concept & AC800M Expert \\
\hline 9 & Instance, Instance, string & AC800M Chemical error \\
\hline 10 & Instance, Instance, string & URAS 3G Public Supply and Utilities CO Messung \\
\hline 11 & Instance, Instance, string & FXE4000 Water and Wastewater error \\
\hline 12 & Instance, Instance, string & FXE4000 Public Supply and Utilities flowmeter \\
\hline 13 & Instance, string, Concept & Chemical H505 Netzwerkmessung Expert \\
\hline 14 & Instance, Instance, Concept & Advance Cemas FTIR Chemical Expert \\
\hline 15 & Instance, Instance, Concept & Limas 11 Education \& Science Configuration \\
\hline 16 & Instance, Instance, Concept & Magnos 106 Chemical Service Report \\
\hline 17 & Instance, Instance, string & Multi-FID 14 Chemical undicht \\
\hline 18 & Instance, Instance, string & Advance Cemas FTIR Chemical Ausfall \\
\hline 19 & Instance, Instance, string & Uras 10P Chemical Wartung \\
\hline 20 & Instance, Instance, string & Uras 14 Chemical Umbau \\
\hline 21 & Instance, Instance, string & Uras 26 Education \& Science störung \\
\hline 22 & Instance, Instance, string, Concept & Advance Cemas FTIR Chemical Wartung Expert \\
\hline 23 & Instance, Instance, string, Concept & Uras 26 Education \& Science störung Expert \\
\hline 24 & Instance, Instance, string, Concept & Uras 14 Chemical Umbau Expert \\
\hline 25 & Instance, Instance, string, Concept & Uras 10P Chemical Wartung Expert \\
\hline
\end{tabular}

\section{Conclusions}

In this paper we have discussed in which ways semantic web technologies can be utilized to provide benefit to industrial service processes. Based on the collected requirements and identified use cases, it provides support to the corresponding roles that depend on knowledge to perform their service tasks.

The underlying architecture is not tied to a particular service domain, but can be applied in other application areas as well. Additionally, a mobile application was outlined to show the flexibility of the described technical framework. Furthermore, various examples regarding the developed service ontology and its positive impact on semantic searches were given.

Vital for the user acceptance were the obtained results from the performed quality and performance evaluations.

\section{Acknowledgements}

This research was funded in part by the German Federal Ministry of Education and Research under grant number 01IA08001G. The responsibility for this publication lies with the authors.

\section{References}

[1] Aleksy, M.; Stieger, B.: Supporting Service Processes with Semantic Mobile Applications. Proceedings of the 8th International Conference on Advances in Mobile Computing and Multimedia (MoMM2010), Paris, France, ACM, 2010, pp. 175-180.

[2] Aleksy, M.; Stieger, B.; Jahnke, T.: Semantic Mobile Applications for Service Process Improvement. International Journal of Business Data Communications and Networking (IJBDCN), IGI Global, Vol. 7, Issue 2, 2011, pp. 1-16.

[3] Aletheia project - Semantic federation of comprehendsive product information. URL: http://www.aletheiaprojekt.de/, 27.07.2011.

[4] Benjamins, V. R., Davies, J., Baeza-Yates, R., Mika, P., Zaragoza, H., Greaves, M., Gomez-Perez, J.M., Contreras, J., Domingue, J., Fensel, D.: Near-Term Prospects for Semantic Technologies. IEEE Intelligent Systems, IEEE Press, Vol. 23, 2008, pp. 76-88.

[5] Delgado, J.; Laplanche, R.; Krishnamurthy, V.: The New Face of Enterprise Search: Bridging Structured and Unstructured Information. Information Management Journal -Prairie Village-, 39(6):40, 2005. 
[6] Demartini, G.: Leveraging Semantic Technologies for Enterprise Search. Proceedings of Conference on Information and Knowledge Management, 2007, pp. 2532.

[7] Fagrell, H.; Forsberg, K.; Sanneblad, J.: FieldWise -A Mobile Knowledge Management Architecture. Proceedings of the ACM 2000 Conference on Computer Supported Cooperative Work, Philadelphia, 2000, pp. 211220.

[8] Grudin, J: Enterprise Knowledge Management and Emerging Technologies, System Sciences. Proceedings of the 39th Annual Hawaii International Conference (HICSS '06), 2006, pp. 57-67.

[9] Halevy, A.J; Ashish, N.; Bitton, D.; Carey, M.; Draper, D.; Pollock, J.; Rosenthal, A.; Sikka, V.: Enterprise information integration: successes, challenges and controversies, Proceedings of the 2005 ACM SIGMOD International Conference on Management of Data, 2005, pp. 778-787.

[10] Kotler, P.: Marketing Management: Millenium Edition. Upper Saddle River, NJ: Prentice-Hall, 2001.

[11] Lovelock, C. H.; Gummesson, E.: Whither Services Marketing?, Journal of Service Research, 7, 2004, pp. 2041.

[12] Makhoul, J.; Kubala, F.; Schwartz, R.; Weischedel, R.: Performance Measures for Information Extraction. In: Proceedings of DARPA Broadcast News Workshop, Herndon, VA, February 1999.

[13] Manning, C.D; Raghavan, P.; Schuetze, H.: Introduction to Information Retrieval, Cambridge University Press, 2008.

[14] Orfali, R.; Harkey, D.; Edwards, J.: Client/Server Survival Guide. John Wiley and Sons, 1999.

[15] PricewaterhouseCoopers: Technology Forecast Spring 2009, 2009.

[16] Shadbolt, N. Berners-Lee, T., Hall, W.: The Semantic Web Revisited. IEEE Intelligent Systems, Vol. 21, No. 3, IEEE Press, 2006, pp. 96-101.

[17] The world factbook: GDP composition by sector. URL:https://www.cia.gov/library/publications/the-worldfactbook/fields/2012.html, 27.07.2011.

[18] van der Spek, R.; Spijkervet, A. Knowlegde Management - Dealing Intelligently with Knowledge, 2005.

[19] W3C: OWL 2 Web Ontology Language Document Overview. URL:http://www.w3.org/TR/owl2-overview/, 28.07.2011.

[20] Wauer, M.; Schuster, D.; Meinecke, J.; Janke, T.; Schill, A.: Aletheia Towards a Distributed Architecture Semantic Federation of Comprehensive Product Information. Proceedings of IADIS International Conference on WWW/Internet, 2009.
[21] Wauer, M.; Schuster, D.; Meinecke, J.; Konzag, A.; Aleksy, M.; Riedel, T.: Semantic Federation of Product Information from Structured and Unstructured Sources, International Journal of Business Data Communications and Networking (IJBDCN), IGI Global, Vol. 7, Issue 2, 2011, pp. 69-97. 